\title{
BMJ Open The health and well-being of Australia's future medical doctors: protocol for a 5-year observational cohort study of medical trainees
}

\author{
Erin Cvejic, ${ }^{1,2}$ Gordon Parker, ${ }^{1,3}$ Samuel B Harvey, ${ }^{1,3,4}$ Zachery Steel, ${ }^{1,3,5}$ \\ Dusan Hadzi-Pavlovic, ${ }^{1}$ Claire L Macnamara, ${ }^{1}$ Uté Vollmer-Conna ${ }^{1}$
}

To cite: Cvejic E, Parker G, Harvey SB, et al. The health and well-being of Australia's future medical doctors: protocol for a 5 -year observational cohort study of medical trainees. BMJ Open 2017;7:e016837. doi:10.1136/ bmjopen-2017-016837

- Prepublication history for this paper is available online. To view these files please visit the journal online (http://dx.doi. org/10.1136/bmjopen-2017016837).

Received 14 March 2017 Revised 21 June 2017 Accepted 4 August 2017

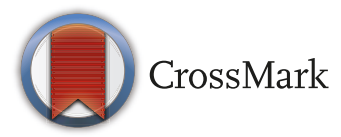

${ }^{1}$ School of Psychiatry, University of New South Wales, Sydney, New South Wales, Australia ${ }^{2}$ The University of Sydney, School of Public Health, Sydney, New South Wales, Australia ${ }^{3}$ Black Dog Institute, Prince of Wales Hospital, Randwick, New South Wales, Australia

${ }^{4}$ St. George Hospital, Kogarah, New South Wales, Australia

${ }^{5}$ Richmond Hospital, St. John of God Health Care, North Richmond, New South Wales, Australia

Correspondence to Uté Vollmer-Conna; ute@unsw.edu.au

\section{ABSTRACT}

Introduction Clinical training in the undergraduate medical course places multiple stressors on trainees, which have been held to lead to heightened distress, depression, suicide, substance misuse/abuse and poor mental health outcomes. To date, evidence for morbidity in trainees is largely derived from cross-sectional surveybased research. This limits the accuracy of estimates and the extent to which predispositional vulnerabilities (biological and/or psychological), contextual triggers and longer-term consequences can be validly identified. Longitudinal clinical assessments embedded within a biopsychosocial framework are needed before effective preventative and treatment strategies can be put in place. Methods and analysis This study is an observational longitudinal cohort study of 330 students enrolled in the undergraduate medicine course at the University of New South Wales (UNSW) Sydney, Australia. Students will be recruited in their fourth year of study and undergo annual assessments for 4 consecutive years as they progress through increasingly demanding clinical training, including internship. Assessments will include clinical interviews for psychiatric morbidity, and self-report questionnaires to obtain health, psychosocial, performance and functioning information. Objective measures of cognitive performance, sleep/activity patterns as well as autonomic and immune function (via peripheral blood samples) will be obtained. These data will be used to determine the prevalence, incidence and severity of mental disorder, elucidate contextual and biological triggers and mechanisms underpinning psychopathology and examine the impact of psychopathology on performance and professional functioning.

Ethics and dissemination Ethics approval has been granted by the UNSW human research ethics committee (reference HC16340). The findings will be disseminated through peer-reviewed publications and conference presentations, and distributed to key stakeholders within the medical education sector. The outcomes will also inform targeted preventative and treatment strategies to enhance stress resilience in trainee doctors.

\section{INTRODUCTION}

The mental health of medical trainees constitutes a significant global problem, as

\section{Strengths and limitations of this study}

- This longitudinal cohort study will deliver substantive clinical insights into the mental health of medical trainees and identify root causes and key biological and psychosocial mechanisms underlying psychopathology in this group.

- The study design enables the longitudinal linking of predisposing vulnerabilities and current mental health status to longer-term health and functional outcomes.

- Active support from the key student body and university medical faculty ensures ongoing endorsement to maximise recruitment and minimise cohort attrition.

- The repeated assessments and data collected at each visit are comprehensive and require a time commitment from participants; however, they provide essential data to map for the first time causal contingencies leading to mental health problems in medical trainees.

- Although recent meta-analytic data indicate that mental health problems in medical trainees are similar globally, recruitment of participants from a single inner city medical school may limit the generalisability of some findings.

demonstrated by accumulating research evidence $^{1-3}$ and a series of publicised suicides. $^{45}$ A recent meta-analysis of 195 studies from 47 countries highlighted that $27 \%$ of medical student respondents screened positive for depression or depressive symptoms, and $11 \%$ reported experiencing suicidal ideation. ${ }^{1}$ These estimates are between two and five times higher than that reported in the general population. Similarly, findings from a nationwide Australian survey ${ }^{6}$ indicated that one in five medical student respondents experienced suicidal thoughts in the preceding 12 months, over half reported emotional exhaustion, and $43 \%$ had a high likelihood of minor psychiatric disorder. 
Such high rates of mental health problems warrant action to better understand the root causes, triggers and vulnerabilities underlying psychopathology in medical trainees.

A career in medicine brings about multiple stressors including excessive workloads and heavy professional responsibility in the context of frequent exposure to disease, death and suffering, ${ }^{7}$ all of which begin during early undergraduate training. While stressful work environments and demanding workloads have a documented impact on mental health and well-being, ${ }^{6-9}$ trainees may carry further vulnerabilities potentiating the risk of maladaptive responses to such stressors. Personality traits of conscientiousness, perfectionism and high neuroticism, which predispose towards unwavering persistence and an inability to relax and relinquish control, have been linked to high levels of stress, emotional exhaustion and mental health impairment in trainees and doctors. ${ }^{6} 71011$ These have serious ramifications beyond the personal level, as they likely impact on medical trainees' long-term health and ability to deliver the best possible medical care to patients in their future careers. ${ }^{12}{ }^{13}$ Furthermore, a series of studies has documented stigmatising attitudes of medical doctors and students in regard to the competency and career opportunities of colleagues with known mental health conditions. ${ }^{6}{ }^{14} 15$ These attitudes often impede trainees and practitioners from engaging in appropriate help-seeking, representing a major barrier to management and recovery.

A number of key questions regarding the mental health of medical trainees remain unanswered. Highquality epidemiological data on the prevalence of mental disorder among medical trainees remain absent, due in part to an almost exclusive reliance on self-report measures and distinct sampling biases (ie, the low response rates may be associated with a higher likelihood of those with morbidity responding to surveys). Indeed, the response rate for Australian medical students in the National Mental Health Survey of Doctors and Medical Students ${ }^{6}$ was very low at $27 \%$; this is unlikely to be fully representative of the medical student population, thus compromising valid estimates of disorder prevalence. ${ }^{16}$ The extent to which mental health problems are pre-existing and the incidence of new disorders over the course of clinical training remain unknown. Additionally, the full spectrum of mental health complaints likely to be seen in a young adult population, including eating disorders, has not yet been examined. Such limitations argue for longitudinal studies employing robust diagnostic assessment and recruitment strategies that maximise sample representativeness and response and retention rates. ${ }^{10}$

Considerable doubt also remains regarding the consequences of poor mental health among medical trainees and junior doctors. Studies of other working populations have shown that mental disorders can have a dramatic impact of workplace performance, even without sickness absence; a phenomena known as 'presenteeism'. ${ }^{17}$ Previous studies have shown that hospital consultants with psychiatric morbidity are more likely to be irritable with patients and provide a lower standard of care. ${ }^{12}$ Direct assessment of the impact of mental health symptoms on cognitive and behavioural functioning has not been reported previously. A clear understanding of the impact of poor mental health on medical trainees and their practice is required before practical steps can be designed to address such problems.

Documenting the biological underpinnings of poor mental health in medical trainees is also critical for early identification and possible intervention, but to date remains commonly overlooked. Mental distress, depression and negative health practices have been associated with poor sleep, autonomic imbalance toward greater stress reactivity as well as disturbed immune functioning, including higher concentrations of inflammatory markers, and suboptimal response to immune challenges. ${ }^{18-21}$ These changes can in turn impact on key central nervous system regulatory pathways, perpetuating poor mental health and suboptimal functioning. ${ }^{22-24}$ Longitudinal monitoring of these biological systems in medical trainees to reveal physiological alterations that may predispose to (or develop concomitant with) psychopathology has not yet been undertaken.

The adoption of a biopsychosocial approach to assessment over the longitudinal course of training is therefore required to extend beyond simple description of the problem towards a more holistic understanding of the root causes, drivers and consequences of psychiatric morbidity in medical trainees. To achieve this, we will conduct a longitudinal cohort study combining gold standard epidemiological methods (to capture valid clinical data) with state-of-the-art biobehavioural study approaches, including the monitoring of biological systems (via quantification of autonomic and inflammatory biomarkers, and recording of 24 hours sleep/wake activity and autonomic patterns) and key behavioural and functioning parameters.

Specifically, the study aims to:

1. determine the prevalence and incidence of mental disorders (including depression, anxiety, eating and substance misuse disorders) among medical trainees across increasingly demanding clinical training years through to internship;

2. elucidate biological mechanisms critical in predisposing to and the emergence and maintenance of psychopathology by monitoring key regulatory systems linked to the stress response (ie, sleep, immune and autonomic functioning);

3. examine the contribution of existing psychosocial risk factors (including exposure to life or trainingrelated adversity, personality and coping styles, and social support) on the development of new triggers emerging in response to the emotional, existential and work place-related challenges met in clinical training; and

4. measure the impact of mental disorder, psychiatric morbidity and associated biological dysfunction on cognitive, academic and work performance. 
It is hypothesised that (1) the prevalence, incidence and severity of mental disorder will increase as trainees transition through increasingly demanding clinical years and internship; (2) disturbances in the major stress-response systems (pre-existing or emerging) will potentiate psychiatric and functional impairment; (3) psychosocial load will additionally impact on the mental health and wellbeing of medical trainees; and (4) medical trainees who develop mental health problems during their training with associated perturbations in key biological systems will show substantial impairment in their cognitive, academic and work performance. Utilising such an approach will maximise the capacity to unlock issues of vulnerability and risk in future doctors, and advance understanding of the aetiology of mental disorders more generally.

\section{METHODS AND ANALYSIS \\ Study design}

This is an observational longitudinal cohort study of $\sim 330$ undergraduate medical students ( $50 \%$ female) enrolled at the University of New South Wales (UNSW), Sydney, Australia. The study will be conducted over a 5-year period.

\section{Sampling and recruitment}

UNSW Sydney has one of the largest medical schools in Australia, with a yearly intake of approximately 275 students ( 1650 total students enrolled across the 6-year Doctor of Medicine (MD) programme). To achieve an initial sample size of at least 330 participants, recruitment will continue over 2 years targeting two consecutive fourth year (Y4) student cohorts, with a conservative targeted recruitment rate of $60 \%$. This study is in a unique position to have the active support of the university Faculty and the medical student representative body (MedSoc), ensuring ongoing endorsement and promotion critical for effective recruitment. From similar cohort studies successfully conducted by members of the research team, ${ }^{25-28}$ an attrition rate of $\leq 20 \%$ is anticipated, leaving a final sample size of approximately 264 medical trainees ( $50 \%$ female). This estimate of attrition is conservative; the combination of the strong and active support for this study from Faculty, MedSoc and the student cohort, maintenance of contact between annual assessments via half-yearly online surveys and reminder emails and a financial reimbursement commensurate with the time commitment involved for follow-up assessments will minimise attrition.

Recruitment for preliminary pilot studies commenced in November 2016. Recruitment for the definitive study will commence once full funding has been obtained. The cohort study will be advertised to all enrolled Y4 students via targeted emails, announcements during lectures and on social media associated with the Faculty and MedSoc. Recruitment will then take place by staggered email invitation, distributed to consecutive waves of 20 randomly selected students. Interested individuals will opt-in to be contacted by a member of the research team for assessment scheduling. The only requirement for inclusion in the study is active enrolment in the fourth year of medical training at UNSW. Participants will be reimbursed $\$ 100$ per annual assessment for their time.

\section{Sample size estimates}

Statistical power estimates were performed for the projected final sample size of 264 students. Based on existing literature ${ }^{126}$ and pilot data collected by our group, we assume an initial prevalence for psychiatric morbidity of $\sim 30 \%$ and increasing incidence of $\sim 10 \%$. The projected sample size will have greater than $80 \%$ power (at $\alpha=0.05$ ) to provide informatively narrow CIs for these rates and to detect differences in the range of 11-19 percentage points both cross-sectionally and over time (hypothesis 1). The sample size will be able to detect effect sizes equivalent to ORs $\geq 2.2$ (Cohen's $d \geq 0.48$ ), corresponding to minimal between-group differences (eg, males vs females) well in line with other studies in this field, and is sufficient to examine factors affecting state or severity through regression models (hypothesis 2 and 3). Further, the projected final sample size will provide $>90 \%$ power to replicate univariate associations between distress, behavioural, biological and performance variables $(r=0.31-0.77)$ as detected in preliminary data (hypothesis 4 ) and $>80 \%$ power for multivariate associations in regression modelling.

\section{Data collection}

Data collection for the definitive study will occur over a 5-year period. Individual participants will undergo a comprehensive assessment annually for 4 consecutive years as they progress through increasingly demanding training (ie, Y4, Y5, Y6, internship). Participants will also be contacted halfway between annual visits to complete a brief online survey to maintain engagement with the study, minimise attrition and obtain additional data on key areas of interest.

\section{Annual assessment protocol}

Consenting participants will be scheduled to attend our laboratory individually on weekday mornings for annual assessments, which take approximately $90 \mathrm{~min}$ to complete (see figure 1). A structured interview will be administered by trained research staff who are not involved in the teaching of medical course content or trainee supervision in any way. Participants will then complete self-report questionnaires to obtain demographic, health, functioning, lifestyle and academic performance information. Autonomic activity will then be recorded at rest, and during the completion of a brief computer-based cognitive assessment. A venous blood sample of approximately $30 \mathrm{~mL}$ will be collected from the participant for storage of serum and plasma. Before departure, participants will be fitted with non-invasive ambulatory monitoring devices to obtain continuous recording of physiological parameters over the next 24 hours (via Equivitial EQ-02 bioharness, Hidalgo, Cambridge, Cambridgeshire, UK), and sleep/ 


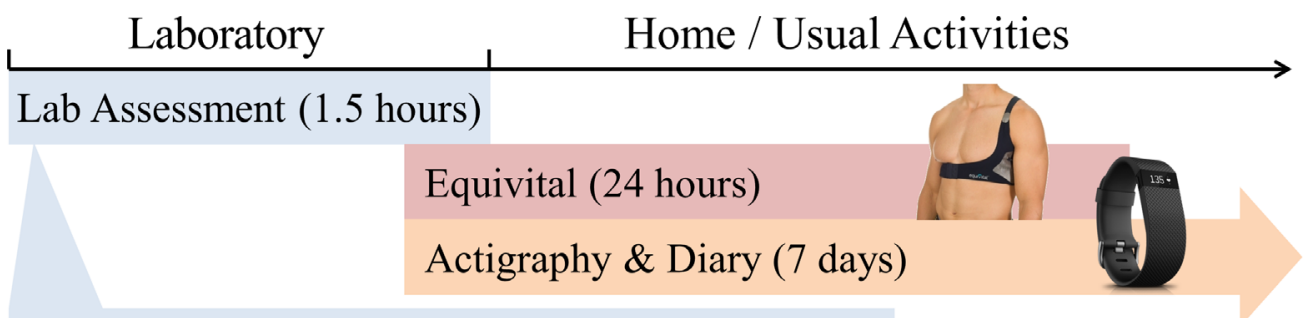

Clinical interview, self-report questionnaires, autonomic \& cognitive assessment, blood sampling

Figure 1 Annual assessments include a 90-min laboratory-based assessment. Ambulatory physiological monitoring for the next 24 hours and 7 days of actigraphy and diarised sleep and mood monitoring are completed alongside regular activities.

activity data over the next 7 days (via Fitbit ChargeHR, Fitbit, San Francisco, California, USA). Ambulatory monitoring takes place alongside regular activities. Participants will also be asked to maintain a sleep/activity/mood log for the next 7 days.

\section{Clinical interview and self-report questionnaires}

A validated structured diagnostic interview (Mini- International Neuropsychiatric Interview; MINI 7.029) will be used to screen for the presence of mood, anxiety, eating, post-traumatic stress and substance-related and addiction disorders. A brief medical history will also be taken.

Standard questionnaires will be used to measure traitbased characteristics of: resilience (Brief Resilience Scale $^{30}$ ), childhood adversity and trauma (Childhood Trauma Questionnaire-Short Form ${ }^{31}$ ) and personality (NEO Five-Factor Personality Inventory ${ }^{32}$ ); and statebased measures of: somatic symptoms (Somatic and Physical Health Report ${ }^{33}$ ), psychological distress (Kessler Psychological Distress Scale; K10), ${ }^{34}$ functional social support (Duke Functional Social Support Questionnaire ${ }^{35}$ ), sleep quality (Pittsburgh Sleep Quality Index ${ }^{36}$ ), diet and nutrition (adapted from PrimeScreen ${ }^{37}$ ), physical activity (International Physical Activity Questionnaire-Short Form ${ }^{38}$ ), alcohol usage (Alcohol Usage Disorders Identification Test ${ }^{39}$ ) and functional impairment (Sheehan Disability Scale; SDS). ${ }^{40}$

Brief questionnaire-based assessments conducted midway between annual visits will be delivered online and include the K10, SDS, and the Screening Questionnaire for Common Mental Disorders. ${ }^{41}$ Annual follow-up assessments will involve a briefer battery of questionnaires assessing only state-based measures.

\section{Academic and cognitive performance measures}

To obtain a rounded assessment of mental performance and functioning, the Health and Workplace Performance Questionnaire $^{42}$ will be used to assess overall vocational performance, absenteeism and presenteeism. Academic performance for students will be indexed by their self-reported Weighted Average Mark, representing academic standing (as cumulative performance) in the medical degree.
Cognitive performance across the domains of verbal and spatial working memory (SWM), motor and processing speed and response inhibition will be assessed using a computerised battery of five tasks. Participants will initially be presented with a list of 20 uncommon words (figure 2A), one at a time for $3 \mathrm{~s}$ each, and asked to remember these words for recall at the end of assessment (delayed word recognition; DWR). Psychomotor vigilance (PVT), ${ }^{43}$ digit-symbol coding (DSC; equivalent to the Digit Symbol Substitution test forming part of the Wechsler Adult Intelligence Scale ${ }^{44}$ ) and SWM tasks will then be presented in randomised order, counterbalanced across participants. The PVT (figure 2B) assesses simple psychomotor response speed over a $3 \mathrm{~min}$ period. The $2 \mathrm{~min}$ DSC requires rapid matching-to-sample responses, from which processing speed and accuracy are obtained (figure 2C). The SWM task requires participants to memorise and reproduce five randomly generated visual sequences of squares illuminating individually on a 4-by-4 grid (figure 2D). Correct recall results in an increase in sequence length, whereas an error triggers a new sequence to commence. The DWR task will then be completed, requiring identification of the 20 words
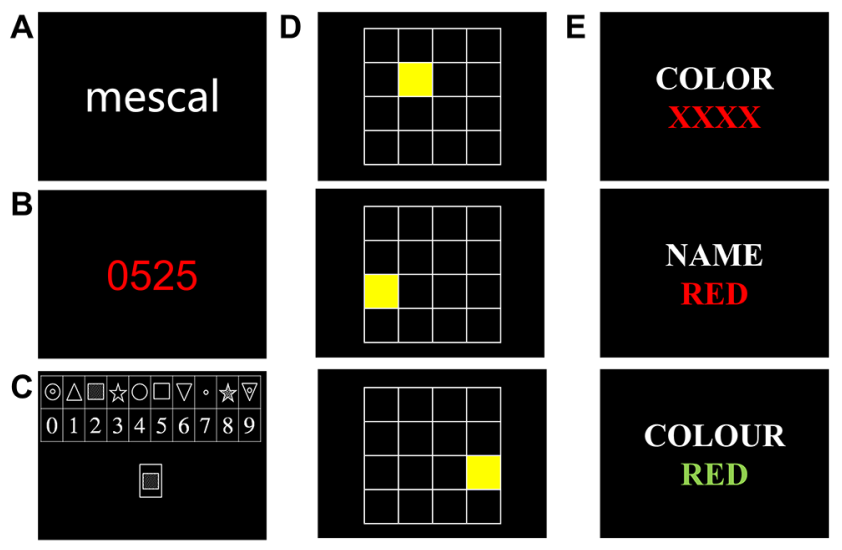

Figure 2 Graphical representation of the computerised cognitive task battery assessing verbal working memory (A), psychomotor response speed and sustained attention (B), matching-to-sample (C), spatial working memory (D) and response inhibition $(\mathrm{E})$. 
initially presented among 20 other new words matched for word use frequency. Finally, a computerised version of the Stroop task ${ }^{45}$ will be presented (figure $2 \mathrm{E}$ ), requiring participants to inhibit prepotent responses by responding to colour-word stimuli on the basis of either the semantic meaning of the word, or the colour in which the word appeared, eliciting measures of both response speed and accuracy. The Stroop task will always be performed last to allow measurement of autonomic reactivity to cognitive challenge to be consistently assessed after comparable testing durations.

\section{Autonomic assessment}

Laboratory-based autonomic measures will include three-lead ECG and respiration (via a strain gauge transducer) recorded at $1 \mathrm{kHz}$ using PowerLab and LabChart Pro (ADInstruments, Bella Vista, New South Wales, Australia). A 10 min baseline recording will be obtained from all participants while seated comfortably in a semireclined position. Autonomic activity will also be recorded continuously throughout cognitive testing to assess reactivity to stressors. Ambulatory autonomic monitoring for a 24-hour period (including during nocturnal sleep) will be achieved via a lightweight bioharness system (Equivital, Hidalgo, Cambridge, Cambridgeshire, UK). The Equivital device consists of a two-channel ECG (sampling rate: $256 \mathrm{~Hz}$ ), respiratory belt $(25.6 \mathrm{~Hz})$, skin temperature sensor $(25.6 \mathrm{~Hz})$ and tri-axial accelerometer $(256 \mathrm{~Hz}$, enabling detection of body orientation and movement), housed in a comfortable chest-worn strap. Obtained data will be extracted and processed using LabChart Pro.

\section{Sleep and activity measures}

At each annual assessment, participants will wear a commercially available activity monitor (Fitbit ChargeHR, Fitbit) on their non-dominant wrist to monitor sleepwake cycle and physical activity levels continuously for 7 consecutive days/nights. A daily sleep/activity diary will be used to record sleep/wake times, sleep quality and mood ratings over the same period, which will assist in the interpretation of actigraphy data. Good reliability of self-reported sleep timing and quality (compared with actigraphy) has been documented. ${ }^{46}$ Weekly averages and variance of sleep duration, bedtime and sleep quality can also be derived.

\section{Blood processing and bioassays}

Blood samples will be collected by a trained and experienced phlebotomist at the same time of day to control for diurnal variations, and processed and stored under strict endotoxin-minimised conditions. Sera/plasma will be stored at $-80^{\circ} \mathrm{C}$ in vapour phase nitrogen until assayed for the cytokines interleukin (IL) $-1 \beta$, IL-6, IL-10, tumour necrosis factor- $\alpha$, and interferon- $\gamma$ (human cytokine 5-plex, Bio-Rad Laboratories, Irvine, California, USA); and for C reactive protein via high-sensitivity ELISA (Invitrogen, Carlsbad, California, USA).

\section{Data analysis plan}

The prevalence and incidence of mental and physical health issues at each time point will be presented with 95\% CIs. The study hypotheses will inform the variables selected for inclusion in models. The contribution of relevant biopsychosocial factors to mental and physical health will initially be explored with univariate regression modelling. The outcome of these analyses will inform the variables for inclusion in multivariate regression and structural equation modelling. Bivariate associations between distress, behavioural and biological variables, as well as performance measures will be explored with Pearson pairwise correlations; the outcome of these analyses will guide relevant variable inclusion in multivariate regression modelling. Only relevant variables (eg, variables showing a univariate association to outcome variables with $\mathrm{p}>0.25$, or are a known biological risk factor for the outcome, and with sufficient variability in the obtained data) will be controlled for in the analyses. Model diagnostics will be performed on all final models to screen for model fit and regression model assumptions. The anticipated samples size will provide $>80 \%$ power to detect small-to-moderate effect sizes with the inclusion of up to eight predictor variables. Differences in the longitudinal dynamics of 24 hours autonomic data will be analysed using linear mixed models (LMM). Under LMM, estimates of effects are based on all available data so that missing data (a degree of which is unavoidable in complex longitudinal datasets) need not be imputed. More complex analyses allowing for cross-sectional integration of biopsychosocial parameters will be achieved by fitting latent-class and factor-analytic models (separately, or jointly in factor-mixture analysis) to identify varyingly defined expressions of wellness (eg, distressed and non-distressed individuals). Structural equation or growth-curve/trajectory models will then be used to explore differences in cross-sectional outcomes to be tracked over time and related to concurrent or prior states and events. Simulations conducted in Mplus for one such projected analysis showed that, for a difference in linear trend between classes equivalent to an effect size $=0.56$ and allowing for subject attrition, statistical power will be $\sim 80 \%$.

\section{ETHICS AND DISSEMINATION}

Potential participants will be informed that their decision of whether or not to participate in the study, and the outcomes of any involvement in the study, will not hinder their relationship with the Faculty or any of the investigators, or impact on their progress in the medical course. Similarly, participants will be encouraged to respond in an open and honest manner, and will be re-assured that their participation and the responses they provide will remain confidential and have no bearing on their involvement in the study, or on their medical course progress. All participants will provide informed written consent prior to commencing any assessments. Participants will also be notified that they are free to with draw from the study at any time without consequence. 
Assessments are non-invasive; however, participants may feel that some of the questions asked are stressful or upsetting. Participants will be informed that if they do not wish to answer a question, they may skip it and go to the next question, or they may stop immediately. Similarly, if participants find any task too demanding or worrying, they are free to withdraw at any time without consequence. Notably, this study has the potential to identify some students who may be experiencing psychological distress within the high-risk range. The study's risk management procedures will be triggered if participants respond in the severe range or indicate suicidal ideation on any of the symptom or distress measures in the assessment battery. In these circumstances, the participant will be contacted by a consultant psychiatrist (who is not directly involved with the study) and, as required, will be referred to the appropriate health services. As this is a duty of care requirement, all participants will be asked to provide consent to be contacted in such an eventuality. If participants would prefer to contact trained mental health professionals external to the university about general feelings of distress, the contact details for a confidential telephone support service will also be provided during the consent process.

All response data will be kept confidential (except as required by the duty of care measure). Participants will be assigned a unique study identification number on enrolment, which will be used across all study materials (questionnaires, computer files and biological samples) allowing data to be linked during analysis without revealing the identity of the individual participants. In all forms of dissemination, only deidentified data will be presented as group means and differences to maintain the anonymity of participants. Data will be retained at the completion of the study for a minimum of 7 years, and stored and disposed of in line with UNSW requirements. All human biospecimen disposals will comply with relevant workplace health and safety and biohazard guidelines (eg, autoclaving and incineration).

The results of this study will be prepared for publication in international peer-reviewed journals and presented at local, national and international conferences. Study updates and interim reports will be made available on the study website. The final report containing summary data will be distributed to all participants, and forwarded to key stakeholders within the medical education and education mental health sectors.

\section{DISCUSSION}

Elevated rates of mental health problems have been reported among medical doctors and trainees in Australia ${ }^{6}$ and internationally, ${ }^{1}$ with clear links demonstrated between the health of medical professionals and the effectiveness of the healthcare they provide. ${ }^{12}{ }^{47}$ Although the importance of mental health problems among doctors has long been recognised, the profession has historically neglected serious consideration of the topic, with medical training tending to reinforce the idea that doctors should be invincible and immune to mental disorders. ${ }^{14}$ To date, no study has included clinical assessments that derive valid estimates of diagnosable mental disorder, combined with objective markers of biological and cognitive functioning to elucidate tangible mechanisms and consequences of poor mental health in medical trainees.

This study will provide the first data-rich investigation mapping longitudinal trajectories of psychiatric morbidity in medical trainees throughout critical stages of their clinical training, by combining gold standard epidemiological clinical assessment with dynamic biological measures linked to stress response systems and assessment of cognitive, academic and workplace performance. The cohort will deliver missing information about the relative importance and functional interactions of significant biological and psychosocial risk and resilience factors (predispositions) with real-life stressors (precipitants) in the emergence and chronicity of mental disorders during critical phases of training. Further, this study will identify risk profiles to psychopathology and inform targeted solutions to enhance stress resilience in trainee doctors. Improving the capacity of doctors to understand their own health issues will enhance their credibility as role models and their ability to provide optimal care to patients.

Acknowledgements The authors wish to acknowledge Emily Jansen, Beryl Lin and Eric Xu (UNSW MedSoc), Professor Anthony O'Sullivan (Programme Authority, UNSW Medicine), Professor Philip Jones (former Associate Dean, Education, UNSW Medicine), Professor Gary Velan (Associate Dean, Education, UNSW Medicine) and the UNSW Faculty of Medicine for their support and valuable assistance with setting up the study, and Dr Jim Lemon for the development of the computerised cognitive tasks.

Contributors EC, GP, SBH, ZS, DH-P, CLM and UV-C conceived and designed the study. EC and CLM acquired preliminary data. DH-P provided statistical advice. EC drafted the manuscript. GP, SBH, ZS, DH-P, CLM and UV-C critically revised the manuscript at each stage. All authors (EC, GP, SBH, ZS, DH-P, CLM and UV-C) have read and approved the final submitted version of the manuscript.

Funding SBH is supported by funding from NSW Health. Seed funding for this work was provided by an Australian National Health and Medical Research Council (NHMRC) Program Grant (\#1037196) awarded to GP. The funding body played no role in the design of the protocol. Additional funding is being sought to commence the definitive study.

Competing interests None declared.

Ethics approval University of New South Wales Human Research Ethics Committee (Reference \#HC16340).

Provenance and peer review Not commissioned; externally peer reviewed.

Open Access This is an Open Access article distributed in accordance with the Creative Commons Attribution Non Commercial (CC BY-NC 4.0) license, which permits others to distribute, remix, adapt, build upon this work non-commercially, and license their derivative works on different terms, provided the original work is properly cited and the use is non-commercial. See: http://creativecommons.org/ licenses/by-nc/4.0/

(c) Article author(s) (or their employer(s) unless otherwise stated in the text of the article) 2017. All rights reserved. No commercial use is permitted unless otherwise expressly granted.

\section{REFERENCES}

1. Rotenstein LS, Ramos MA, Torre M, et al. Prevalence of depression, depressive symptoms, and suicidal ideation among 
medical students: a systematic review and meta-analysis. JAMA 2016;316:2214-36.

2. Mata DA, Ramos MA, Bansal N, et al. Prevalence of depression and depressive symptoms among resident physicians: a systematic review and meta-analysis. JAMA 2015;314:2373-83.

3. Harvey SB, Laird B, Henderson M, et al; The mental health of health care professionals: A review for the Department of Health. London, UK: National Clinical Assessment Service, 2009.

4. Goldman ML, Shah RN, Bernstein CA. Depression and suicide among physician trainees: recommendations for a national response. JAMA Psychiatry 2015;72:411-2.

5. Rubin R. Recent suicides highlight need to address depression in medical students and residents. JAMA 2014;312:1725-7.

6. beyondblue. National mental health survey of doctors and medical students. Victoria, Australia: Beyond Blue Limited, 2013.

7. Riley GJ. Understanding the stresses and strains of being a doctor Med J Aust 2004:181:350-3.

8. Henderson M, Harvey SB, Overland S, et al. Work and common psychiatric disorders. J R Soc Med 2011;104:198-207.

9. Stansfeld S, Candy B. Psychosocial work environment and mental health--a meta-analytic review. Scand J Work Environ Health 2006:32:443-62.

10. Elliot L, Tan J, Norris S. The mental health of doctors: A systematic literature review. Victoria, Australia: Beyond Blue Limited, 2010.

11. Guille $\mathrm{C}$, Clark $\mathrm{S}$, Amstadter AB, et al. Trajectories of depressive symptoms in response to prolonged stress in medical interns. Acta Psychiatr Scand 2014:129:109-15.

12. Taylor C, Graham J, Potts $\mathrm{H}$, et al. Impact of hospital consultants poor mental health on patient care. Br J Psychiatry 2007;190:268-9.

13. Oberg EB, Frank E. Physicians' health practices strongly influence patient health practices. J R Coll Physicians Edinb 2009;39:290-1.

14. Henderson M, Brooks SK, Del Busso L, et al. Shame! Selfstigmatisation as an obstacle to sick doctors returning to work: a qualitative study. BMJ Open 2012;2:e001776.

15. Cheng DR, Poon F, Nguyen TT, et al. Stigma and perception of psychological distress and depression in Australian-trained medical students: results from an inter-state medical school survey. Psychiatry Res 2013;209:684-90.

16. Knudsen AK, Hotopf M, Skogen JC, et al. The health status of nonparticipants in a population-based health study: the Hordaland Health Study. Am J Epidemiol 2010;172:1306-14.

17. Harvey SB, Glozier N, Henderson M, et al. Depression and work performance: an ecological study using web-based screening. Occup Med 2011;61:209-11.

18. Lucas M, Chocano-Bedoya P, Schulze MB, et al. Inflammatory dietary pattern and risk of depression among women. Brain Behav Immun 2014;36:46-53.

19. Kemp AH, Quintana DS. The relationship between mental and physical health: insights from the study of heart rate variability. Int $J$ Psychophysiol 2013;89:288-96.

20. Smith $A$, Vollmer-Conna $U$, Bennett $B$, et al. The relationship between distress and the development of a primary immune response to a novel antigen. Brain Behav Immun 2004;18:65-75.

21. Smith AJ, Vollmer-Conna U, Bennett B, et al. Influences of distress and alcohol consumption on the development of a delayed-type hypersensitivity skin test response. Psychosom Med 2004;66:614-9.

22. Beaumont $A$, Burton AR, Lemon J, et al. Reduced cardiac vagal modulation impacts on cognitive performance in chronic fatigue syndrome. PLoS One 2012;7:e49518.

23. Gunaratne P, Lloyd AR, Vollmer-Conna U. Mood disturbance after infection. Aust N Z J Psychiatry 2013;47:1152-64.

24. Raison CL, Miller AH. Malaise, melancholia and madness: the evolutionary legacy of an inflammatory bias. Brain Behav Immun 2013;31:1-8.
25. Wilhelm K, Parker G, Dewhurst-Savellis J, et al. Psychological predictors of single and recurrent major depressive episodes. $J$ Affect Disord 1999;54:139-47.

26. Vollmer-Conna U, Cvejic E, Granville Smith I, et al. Characterising acute coronary syndrome-associated depression: Let the data speak. Brain Behav Immun 2015;48:19-28.

27. Piraino B, Vollmer-Conna U, Lloyd AR. Genetic associations of fatigue and other symptom domains of the acute sickness response to infection. Brain Behav Immun 2012;26:552-8.

28. Steel Z, Momartin S, Silove D, et al. Two year psychosocial and mental health outcomes for refugees subjected to restrictive or supportive immigration policies. Soc Sci Med 2011;72:1149-56.

29. Sheehan DV, Lecrubier Y, Sheehan KH, et al. The Mini-International Neuropsychiatric Interview (M.I.N.I.): the development and validation of a structured diagnostic psychiatric interview for DSM-IV and ICD10. J Clin Psychiatry 1998;59(Suppl 20):22-33.

30. Smith BW, Dalen J, Wiggins K, et al. The brief resilience scale: assessing the ability to bounce back. Int J Behav Med 2008; 15:194-200.

31. Bernstein D, Fink L. Manual for the Childhood Trauma Questionnaire. New York, USA: The Psychological Corperation, 1998.

32. Costa PT, McCrae RR. NEO Inventories. Professional Manual. Florida, USA: PAR, 2010.

33. Hadzi-Pavlovic D, Hickie IB, Wilson AJ, et al. Screening for prolonged fatigue syndromes: validation of the SOFA scale. Soc Psychiatry Psychiatr Epidemiol 2000;35:471-9.

34. Kessler RC, Andrews G, Colpe LJ, et al. Short screening scales to monitor population prevalences and trends in non-specific psychological distress. Psychol Med 2002;32:959-76.

35. Broadhead WE, Gehlbach SH, de Gruy FV, et al. The Duke-UNC Functional Social Support Questionnaire. Measurement of social support in family medicine patients. Med Care 1988;26:709-23.

36. Buysse DJ, Reynolds CF, Monk TH, et al. The Pittsburgh Sleep Quality Index: a new instrument for psychiatric practice and research. Psychiatry Res 1989;28:193-213.

37. Rifas-Shiman SL, Willett WC, Lobb R, et al. PrimeScreen, a brief dietary screening tool: reproducibility and comparability with both a longer food frequency questionnaire and biomarkers. Public Health Nutr 2001:4:249-54.

38. Craig CL, Marshall AL, Sjöström M, et al. International physical activity questionnaire: 12 -country reliability and validity. Med Sci Sports Exerc 2003;35:1381-95.

39. Saunders JB, Aasland OG, Babor TF, et al. Development of the Alcohol Use Disorders Identification Test (AUDIT): WHO Collaborative Project on Early Detection of Persons with Harmful Alcohol Consumption-II. Addiction 1993;88:791-804.

40. Sheehan DV, Harnett-Sheehan K, Raj BA. The measurement of disability. Int Clin Psychopharmacol 1996;11(Suppl3):89-95.

41. Donker T, van Straten A, Marks I, et al. A brief Web-based screening questionnaire for common mental disorders: development and validation. J Med Internet Res 2009;11:e19.

42. Kessler RC, Barber C, Beck A, et al. The World Health Organization Health and Work Performance Questionnaire (HPQ). J Occup Environ Med 2003;45:156-74.

43. Basner M, Mollicone D, Dinges DF. Validity and Sensitivity of a Brief Psychomotor Vigilance Test (PVT-B) to Total and Partial Sleep Deprivation. Acta Astronaut 2011;69:949-59.

44. Wechsler D. Wechsler Adult Intelligence Scale. 4th ed. San Antonio TX: Perason Assessment, 2008.

45. Stroop JR. Studies of interference in serial verbal reactions. J Exp Psychol 1935;18:643-62.

46. Kawada T. Agreement rates for sleep/wake judgments obtained via accelerometer and sleep diary: a comparison. Behav Res Methods 2008;40:1026-9.

47. Boorman S. NHS health and well-being: final report. London, UK: Department of Health, 2009. 